\title{
Retinoic Acid: A Potential Risk Factor for Congenital Heart Disease
}

Maria Fareed Siddiqui*I, Sehar Farooq ${ }^{2}$, Amna Komal Khan ${ }^{3}$, Tallat Anwar Faridi ${ }^{4}$, Ayaz Ali Khan ${ }^{1}$

1. Department of Pharmacy, Faculty of Pharmacy, University of Lahore, Pakistan

2. Institute of Molecular Biology and Biotechnology, University of Lahore, Pakistan

3. Department of Biotechnology, Kinnaird College for Women, Lahore, Pakistan

4. University Institute of Physical Therapy, University of Lahore, Lahore, Pakistan

* Corresponding Author's Email: *maria.pharmacist@gmail.com, maria.siddiqui@pharm.uol.edu.pk

ABSTRACT: Retinoic acid (RA), a derivative of vitamin A, plays an essential role in human beings during growth and development. Intake of vitamin $A$ in body is by either animal/plant diet or use of medication to treat skin problems and cancer. Adequate intake of retinoic acid is critical as high and low quantities of retinoid lead to developmental defect by acting through retinoid receptors present in human body, which actively modulate many signaling pathways and control embryonic development and differentiation. Excess intake of RA cause congenital heart diseases (CHD) by up and down regulation of genes associated with CHD. Therefore, intake of retinoic acid or other analogous drugs especially during pregnancy enhances the risk of developing CHD. Physician and pharmacist should provide proper counseling to female patients about the benefits and risks of drugs prescribed during pregnancy. Furthermore, advancement in therapies likes surgery, angioplasty and tissue engineering used for the treatment of CHD. This review discusses an association between retinoic acid and $C H D$, its prevention and treatment options available.

Keywords: Congenital heart defect, birth defects, retinoic acid, retinoid receptors,

\section{INTRODUCTION}

Vitamin A (retinol) plays an essential role in human beings that supports to control the cellular differentiation of epithelial tissue. Vitamin A exists in two dietary forms; one is found in animal products and second in plants as carotenoids (Niwa, 2018). They are essential biomolecules for embryonic development maintenance of body homeostasis. Many processes like cellular and epithelial growth, immune response, apoptosis, some nuclear receptors mechanisms and functions of retinoic acid receptor and retinoid $\mathrm{X}$ receptor (RXR) are influenced by retinoids (Perl et al., 2019). 
Throughout early embryonic development, all-trans retinoic acid (atRA), are actively involved in development of pancreas, formation of body axis, cardiogenesis, vision process, breathing functions and neurogenesis. Additional intake of vitamin A results in teratogenisis and chances of birth defects in developing embryo. Intake of vitamin $\mathrm{A}$ and retinoids in excess is dangerous for pregnancy as it causes different anomalies to fetuses' central nervous system, skulls, limbs, face and eyes (Perl and Waxman, 2019). Both high and low levels of retinoic acid (RA) is a predominant risk factor for congenital heart defects with spectrum of developmental defects (Pace et al., 2018). Over intake of RA in the form of medications could contribute towards the development of CHD mostly due to lack of awareness. Henceforth, in this review, we have discussed the importance of adequate intake of RA and its role in the development of CHD and possible treatment modalities for CHD.

\section{Intake of Retinoic acid}

Retinoids used for the treatment of skin diseases like acne, scars, sun damage, wrinkling, skin tone problems, anti-aging as well as breast and ovarian cancer. In US, four retinoids introduced to fulfill the dermatological problems out of which one is 13-cis-retinoic acid isotretinoin (Accutane) used to treat acne.
Use of this medicine showed negative impact on pregnant women. In US, some medicines are strictly ban during pregnancy including accutane and tegison. Exposure to 13-cis-retinoic acid bases cause deformities in developing fetuses and central nervous system deformities. Vitamin A known for its wider role in skin nutrition as enhancer since 1940s stimulates the new cell growth in skin. Early researches focused on the consumption of vitamin A orally but it was associated with some side effects like liver damage, hair loss and softening of skull (Dmitrovsky and Spinella, 2017).

\section{Cellular metabolism of Retinoic Acid \\ Retinoic acid derived from} vitamin A has specific role in organogenesis and cell growth. When cells need retinoic acid, they up-take retinol and convert it into retinaldehyde and retinoic acid. Two important enzymes catalyze this reversible reaction. Retinol dehydrogenases (ROLDH) convert retinol into retinaldehyde and retinal dehydrogenases (RALDH) convert retinaldehyde into retinoic acid. Active metabolites of retinoic acid are, all-Trans-RA, 9-cis-RA, and 4-oxoRA. Retinoic acid serves as a ligand in nucleus and brings transcriptional changes through two families of receptors i.e., retinoic acid receptors 
and retinoid $\mathrm{X}$ receptors (RXR) as

described in fig. 1 (Fadel et al., 2020).

Retinol

Dehydrogenase

Retinal

Dehydrogenase

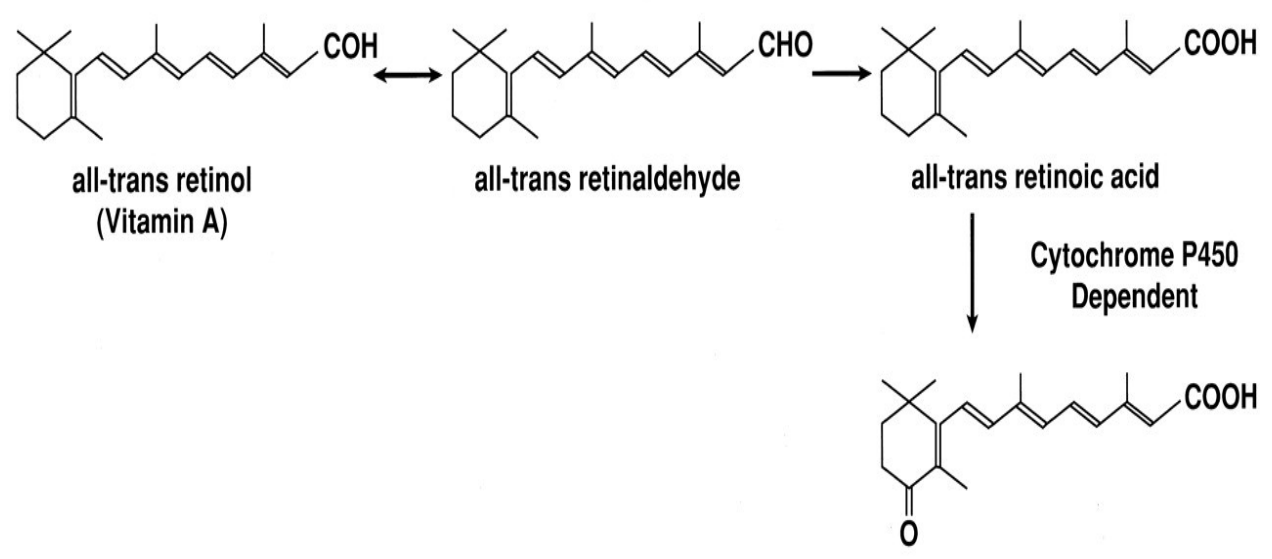

4-oxo all-trans retinoic acid

Fig. 1. Cellular Mechanism of Retinoic Acid (Fadel et al., 2020)

\section{Retinoic acid as a risk factor of Congenital Heart Disease}

Physiological concentrations of retinoic acids regulate the normal growth of embryo and cardio genesis (Wang et al., 2019). In vertebrate's development, congenital malformation occurs in many organs due to increased or decreased level of embryonic RA (Diaz et al., 2020). Some retinoid receptors are actively involved in many signaling pathways and regulate embryonic development \& differentiation processes. Studies on animal models such as vitamin A deficient rat embryos resulted in heart developmental defects including aortic arch and ventricular outflow tract malformations (Wilson et al., 1949). Deficiency or excess of vitamin A mainly affects the valves or membranous septa of heart. It is thought that defect occur in development due to the up and down regulation of specific genes. Due to shortage of retinol certain genes repressed therefore, they not expressed properly. Hox gene is responsible for human embryonic development mainly in fourth week of pregnancy (Piersma et al., 2017). Altered function of this gene due to any mutation and loss of genetic control distorts the normal physiology. In humans, there is loss of CYP26A1 gene related with DiGeorge and Klipplel-Feil syndromes, which involves outflow tract defects (Pennimpede et al., 2010). Excess RA promotes the genetic expression of STRA6, LRAT, DHRS3, CRBP1, CRABP2, and CYP26A1, while it down regulate RBP4, RDH10 and ALDH1A2 expression (Amengual et al., 2014; Billings et al., 2013; Sandell et al., 2012). 
This alters the specification of cardiovascular tissues during early development, left/right decisions and cardiac situs, anteroposterior patterning of the early heart, endocardial cushion formation, and the neural crest (Pan et al., 2007).

\section{Treatments for Congenital Heart Disease}

Many people get complete cure through surgery or medications. Most commonly prescribed medications are diuretics, beta-blockers, digitalis and anticoagulants.

Tet Spell: Tet Spell treated by using beta-blocker using Propranolol but in acute condition treatment with morphine or intranasal fentanyl and a vasopressor like phenylephrine or nor epinephrine are recommended to increase the vascular resistance. Oxygen plays a very efficient role in treating spells. It can help in movement of blood through lungs by decreasing the pressure of deoxygenated blood from the right to left ventricle with the help of VSD (Tsze et al., 2014).

\section{Total Surgical Repair:}

Tetralogy of Fallot treated with surgery to improve the blood flow towards lungs to make sure the correct direction of oxygenated and deoxygenated blood.

Stem Cell Therapy: is a potential treatment for difficult to treat disease. The basic aim is to stimulate the renewal of damaged tissues through stem cell therapy. Various injections depending on type of tissues and diseases recommended for this purpose. Different routes of injection administration like intracoronary, intravenous, and intramyocardial can be used for proper treatment (Brown et al., 2020).

Tissue Engineering: This technique is useful for the renewal of impaired tissue and is most effective in repair of damage or any organ like skeletal muscle, trachea and esophagus not present since birth (Londono et al., 2015). Tissue engineering reached to success in 1994 for the reproduction of tracheal cartilage. Some other techniques like bioengineering used to treat heart defects. If the stem cell embedded in extracellular matrix in the form of prosthetic graft and patches then it works more efficiently. Once the implantation is done in defective heart it alters or grow fast in physiological order (Ott et al., 2011).

Valve Replacement: Heart valves replaced with tissues or mechanical valve also offer an effective treatment of the damaged valves.

Balloon Angioplasty: Balloon angioplasty of pulmonary or aortic vein (BPV, BAV) is the best way for the treatment of congenital heart defects. Balloon angioplasty is a method of surgery in innate narrowing of aortic arch and re-narrowing of the aorta after an operation (Ammar, 2012). When there is narrowing in any part of heart's circulation system, balloon angioplasty is required which is also termed as balloon dilation. Balloon angioplasty helps in improving blood flow by opening the narrowed area of heart. Catheter, a thin 
tube inserted at the top of leg into blood vessels. The tip of catheter inserted into the heart with a balloon, once the balloon reaches at the right place; it helps in opening the narrowed area. The balloon stretches the wall of artery and forces the narrow area to open. When balloon deflates, the catheters are taken out, whilst the injured part of leg is covered with bandage (Byrne et al., 2017).

\section{CONCLUSION}

Vitamin A (retinol) plays an essential role in human beings that supports to control the cellular differentiation of epithelial tissue. Intake of retinol occurs in the form of retinoids when administered to treat skin diseases like acne, scars, sun damage, wrinkling, skin tone problems, anti-aging as well as breast and ovarian cancer. Physiological concentrations of retinoic acids regulate the normal growth of embryo and cardiogenesis. Deficiency or excess of vitamin A mainly affects the valves or membranous septa of heart hence development of congenital heart defects. Retinoic acid is a non-genetic cause of $\mathrm{CHD}$, which leads to the disturbance in any portion of heart, disturbed blood flow, blood flow in wrong direction or complete blockage. Intake of retinoic acid or other drugs during pregnancy enhances the risk of developing CHD. Hence, pregnant women should avoid taking any painkillers, drugs and alcohol. Good control of blood sugar, proper vaccination and complete regular checkup must be promoted. Some chronic condition like epilepsy requires the use of medications during pregnancy. Physician and pharmacist should provide proper counseling to female patients about the benefits and risks of drugs prescribed during pregnancy. Use of multivitamins and folic acid reduce the risk of birth defect in brain and heart (van der Bom et al., 2011). Food and Drug Administration has taken steps such as placing strict risk management protocols on the use of retinoids such as etretinate and isotretinoin, in order to prevent teratogenic defects (Abroms et al., 2006). Furthermore, advancement in therapies like surgery, angioplasty and tissue engineering have helped treat CHD. In depth understanding of underlying mechanism of role of RA in congenital heart disease in animal models and human subjects will help in reducing the risk factor.

\section{REFERENCES}

1. Abroms L, Maibach E, Lyon-Daniel $\mathrm{K}$, Feldman SR (2006). What is the best approach to reducing birth defects associated with isotretinoin? PLoS Med, 3: e483.

2. Amengual J, Zhang N, Kemerer M, Maeda T, Palczewski K, Von Lintig J (2014). STRA6 is critical for cellular vitamin A uptake and homeostasis. Hum. Mol. Genet. 23: 5402-5417.

3. Ammar RI (2012). Balloon Angioplasty for Native Aortic Coarctation in Children and Infants Younger Than 12 Mondis: 
Immediate and Medium-Term Follow-Up. J Invasive Cardiol, 24: 662.

4. Billings SE, Pierzchalski K, Tjaden NE, Pang XY, Trainor PA, Kane MA, Moise AR (2013). The retinaldehyde reductase DHRS3 is essential for preventing the formation of excess retinoic acid during embryonic development. FASEB J. 27: 4877-4889.

5. Brown MA, Rajamarthandan S, Francis B, O'Leary-Kelly MK, Sinha P (2020). Update on stem cell technologies in congenital heart disease. J. Card. Surg. 35: 174-179.

6. Byrne RA, Stone GW, Ormiston J, Kastrati A (2017). Coronary balloon angioplasty, stents, and scaffolds. Lancet. 390: 781-792.

7. Diaz OE, Xue S, Luo X, Nava J, Appelblom A, Morales RA, Das S, Villablanca EJ (2020). Retinoic acid induced cytokines are selectively modulated by liver $\mathrm{X}$ receptor activation in zebrafish. Reprod. Toxicol. 93: 163-168.

8. Dmitrovsky E, Spinella M (2017). Retinoids. In Cancer Therapeutic Targets (pp. 1039-1046): Springer New York.

9. Fadel L, Reho B, Volko J, Bojcsuk D, Kolostyak Z, Nagy G, Muller G,
Simandi Z, Hegedus E, Szabo G, Toth K (2020). Agonist binding directs dynamic competition among nuclear receptors for heterodimerization with retinoid $\mathrm{X}$ receptor. J. Biol. Chem. 295: 10045-10061.

10. Londono R, Badylak SF (2015). Regenerative Medicine Strategies for Esophageal Repair. Tissue Eng. Part B Rev. 21: 393-410.

11. Niwa K (2018). Adult congenital heart disease with pregnancy. Korean Circ. J. 48: 251.

12. Ott LM, Weatherly RA, Detamore MS (2011). Overview of Tracheal Tissue Engineering: Clinical Need Drives the Laboratory Approach. Ann. Biomed. Eng. 39: 2091-2113.

13. Pace ND, Oster ME, Forestieri NE, Enright D, Knight J, Meyer RE (2018). Sociodemographic factors and survival of infants with congenital heart defects. Pediatrics. 142.

14. Pan J, Baker KM (2007). Retinoic acid and the heart. Vitam. Horm. 75: 257-283.

15. Pennimpede $\mathrm{T}$, Cameron $\mathrm{D}$, MacLean G, Li H, Abu-Abed S, Petkovich M (2010). The role of CYP26 enzymes in defining appropriate retinoic acid exposure 
during embryogenesis. Birth Defects Res. Clin. Mol. Teratol. 88: 883-894.

16. Perl E, Waxman JS (2019). Reiterative mechanisms of retinoic acid signaling during vertebrate heart development. J. Dev. Biol. 7: 11.

17. Piersma AH, Hessel EV, Staal YC (2017). Retinoic acid in developmental toxicology: teratogen, morphogen and biomarker. Reprod. Toxicol. 72: 5361.

18. Sandell LL, Lynn ML, Inman KE, McDowell W, Trainor PA (2012). RDH10 oxidation of Vitamin A is a critical control step in synthesis of retinoic acid during mouse embryogenesis. PloS one. 7: e30698.

19. Tsze DS, Vitberg YM, Berezow J, Starc TJ, Dayan PS (2014). Treatment of tetralogy of Fallot hypoxic spell with intranasal fentanyl. Pediatrics, 134: e266e269.

20. Wang S, Moise AR (2019). Recent insights on the role and regulation of retinoic acid signaling during epicardial development. Gen. 57: e23303.

21. Wilson JG, Warkany J (1949). Aortic-arch and cardiac anomalies in the offspring of vitamin A deficient rats. American J. Anat. 85: 113-155. 